\title{
Helium bubble formation in ultrafine and nanocrystalline tungsten under different extreme conditions
}

\author{
O. El-Atwani ${ }^{1,2,3,4}$, K. Hattar ${ }^{5}$, J.A. Hinks, ${ }^{6}$ G. Greaves, ${ }^{6}$ S.S.Harilal ${ }^{1,4}$ and A. Hassanein ${ }^{1,4}$ \\ ${ }^{1}$ School of Nuclear Engineering, Purdue University, West Lafayette, IN 47907 \\ ${ }^{2}$ School of Materials Engineering, Purdue University, West Lafayette, IN 47907 \\ ${ }^{3}$ Birck Nanotechnology Center, West Lafayette, IN 47907 \\ ${ }^{4}$ Center of Materials Under Extreme Environment, West Lafayette, IN 47907 \\ ${ }^{5}$ Department of Radiation Solid Interactions, Sandia National Laboratories, Albuquerque, NM 87185 \\ ${ }^{6}$ School of Computing and Engineering, University of Huddersfield, HD1 3DH, United Kingdom
}

\begin{abstract}
We have investigated the effects of helium ion irradiation energy and sample temperature on the performance of grain boundaries as helium sinks in ultrafine grained and nanocrystalline tungsten. Irradiations were performed at displacement and non-displacement energies and at temperatures above and below that required for vacancy migration. Microstructural investigations were performed using Transmission Electron Microscopy (TEM) combined with either in-situ or ex-situ ion irradiation. Under helium irradiation at an energy which does not cause atomic displacements in tungsten $(70 \mathrm{eV})$, regardless of temperature and thus vacancy migration conditions, bubbles were uniformly distributed with no preferential bubble formation on grain boundaries. At energies that can cause displacements, bubbles were observed to be preferentially formed on the grain boundaries only at high temperatures where vacancy migration occurs. Under these conditions, the decoration of grain boundaries with large facetted bubbles occurred on nanocrystalline grains with dimensions less than $60 \mathrm{~nm}$. We discuss the importance of vacancy supply and the formation and migration of radiation-induced defects on the
\end{abstract}


performance of grain boundaries as helium sinks and the resulting irradiation tolerance of ultrafine grained and nanocrystalline tungsten to bubble formation.

\section{Introduction}

UltraFine Grained (UFG) and NanoCrystalline (NC) metals have been proposed as radiation tolerant materials due to their high grain-boundary area. ${ }^{1}$ The grain boundaries act as defect $\operatorname{sinks}^{2,3}$ with large-angle grain boundaries (angles $>15^{\circ}$ ) being particularly efficient sinks. ${ }^{4}$ Furthermore, recent work has suggested grain boundaries can facilitate Frenkel pair recombination and thus annihilation. ${ }^{5}$ Tungsten is an important material for nuclear fusion applications due to its physical properties ${ }^{6}$ but several irradiation studies have demonstrated considerable drawbacks due to the development of surface morphology when exposed to moderately-high helium doses. ${ }^{7,8,9}$ The use of UFG and NC tungsten with high-angle grain boundaries is one of the proposed solutions to mitigate helium-induced radiation damage. ${ }^{10}$ These materials have been shown also to possess improved mechanical properties compared to commercial coarse-grained tungsten. ${ }^{11,12}$ In addition to being interstitial and vacancy sinks, grain boundaries in tungsten can trap helium during irradiation ${ }^{13}$ and can thus reduce the rate of helium accumulation within the grains themselves. ${ }^{14}$ If the observed surface morphology changes ${ }^{15,8}$ depend on helium bubble formation as proposed in the literature, ${ }^{16}$ then engineering of grain boundary density could be a vital tool for controlling this deleterious phenomenon. It should be mentioned, however, that degradation of mechanical properties (for example, reduced creep resistance $^{17}$ or enhanced grain boundary grooving ${ }^{15}$ ) is likely to occur due to large bubble formation on the grain boundaries. 
The formation of UFG and NC tungsten materials with elongated grains is achievable through several Severe Plastic Deformation (SPD) techniques. ${ }^{11,18}$ Although their use on industrial scales remains a challenge due to limitations on the throughput achievable using current manufacturing technologies, SPD techniques can fabricate high-quality samples for important fundamental studies to gain improved understanding of physical phenomena in these materials. Whilst some theoretical studies ${ }^{5,19}$ have demonstrated the improved radiation resistance of materials with grain boundaries, further experimental studies are crucial to validate these proposed models.

Recently, Bai et al. ${ }^{5}$ demonstrated the effect of grain boundaries as defect sinks. It was shown that grain boundaries absorb interstitial defects and can then annihilate nearby vacancies by reemitting the interstitial atoms back into the grain. Sefta et al. ${ }^{19}$ used molecular dynamics to demonstrate the role of grain boundaries as helium trapping sites. In that work, the introduction of a single grain boundary was shown to result in the trapping of significantly more helium than a single crystal of tungsten.

Fundamental understanding can be acquired through studies in which the irradiation and observation of the dynamic response of a material take place simultaneously. The work reported here involved Transmission Electron Microscopy (TEM) characterization of both in-situ and exsitu helium irradiated UFG and NC tungsten at different ion energies to control atomic displacements and different temperatures to control vacancy migration. Observation of bubble formation and evolution has given invaluable insights into the role of grain boundaries in this technologically important material. 


\section{Experimental}

The formation of UFG and NC tungsten was performed via orthogonal machining as detailed previously. ${ }^{18}$ The TEM samples were produced by electrochemical jet polishing with $0.5 \%$ $\mathrm{NaOH}$ aqueous solution at Room Temperature (RT). No significant variation in mass-thickness contrast was observed between adjacent grains in the TEM samples suggesting negligible preferential etching due to crystallographic orientation and/or grain size.

In-situ TEM during ion implantation was performed using the Microscope and Ion Accelerator for Materials Investigations (MIAMI) facility at the University of Huddersfield which is described in detail elsewhere ${ }^{14}$ and at Sandia National Laboratories' (SNL) new in-situ ion irradiation TEM facility. ${ }^{20}$ Ex-situ ion irradiation followed by TEM characterization were performed using the Interaction of Materials with Particles And Components Testing $(\text { IMPACT })^{21}$ facility in the Center of Materials Under eXtreme Environments (CMUXE) at Purdue University.

Two samples were irradiated in-situ whilst under TEM observation. One sample was irradiated at SNL at RT with $8 \mathrm{keV}$ helium with an angle of $15^{\circ}$ between the ion beam and the sample surface in a JEOL JEM-2100 TEM operating at $200 \mathrm{kV}$. The other sample was irradiated at the MIAMI facility at $1223 \mathrm{~K}$ with $2 \mathrm{keV}$ helium at an angle of $60^{\circ}$ to the sample surface in a 
JEOL JEM-2000FX TEM operating at $200 \mathrm{kV}$. The ion fluxes were $\sim 3.3 \times 10^{16}$ and $1.2 \times 10^{17}$ ions. $\mathrm{m}^{-2} \mathrm{~s}^{-1}$ in the $2 \mathrm{keV}$ and $8 \mathrm{keV}$ experiments, respectively. The range of helium normal to the surface was calculated to be $23.6 \mathrm{~nm}$ (maximum $\approx 70 \mathrm{~nm}$ ) and $10.6 \mathrm{~nm}$ (maximum $\approx 30 \mathrm{~nm}$ ) for the 8 and $2 \mathrm{keV}$ irradiation conditions, respectively, using the Stopping Range of Ions in Matter $(\mathrm{SRIM})^{22}$ Monte Carlo computer code. Two further samples were irradiated ex-situ at the IMPACT facility with $70 \mathrm{eV}$ helium ions at $\mathrm{RT}$ and at $1173 \mathrm{~K}$ both at normal incidence. The ion flux in the $70 \mathrm{eV}$ experiments was $\sim 1 \mathrm{x} 10^{19}$ ions. $\mathrm{m}^{-2} \cdot \mathrm{s}^{-1}$. The range in the $70 \mathrm{eV}$ experiments was calculated to be $1.4 \mathrm{~nm}$ (maximum $\approx 4 \mathrm{~nm}$ ) using SRIM. Irradiation conditions are summarized in Table 1. Post-irradiation, samples were examined using an FEI Titan 80/300 field emission TEM and/or a JEOL JEM-3010 $\mathrm{LaB}_{6}$ TEM both operated at $300 \mathrm{kV}$. Electron BackScattered Diffraction (EBSD) was performed on non-irradiated electrochemically polished samples using an FEI XL40 field emission scanning electron microscope equipped with an EBSD detector.

Table1: Irradiation conditions used in the four experimental regimes (see Fig 2) compared in this study.

\begin{tabular}{|c|c|c|c|c|}
\hline \multirow{2}{*}{$\begin{array}{l}\text { Regime } \\
\text { (see Fig. } \\
\text { 2) }\end{array}$} & \multirow{2}{*}{$\begin{array}{c}\text { Sample } \\
\text { temperature } \\
(K)\end{array}$} & \multicolumn{3}{|c|}{ Helium ion irradiation } \\
\hline & & $\begin{array}{c}\text { Energy } \\
(\mathrm{eV})\end{array}$ & $\stackrel{\text { Flux }}{\left(\text { ions. } \mathrm{m}^{-2} \mathrm{~s}^{-1} \text { ) }\right.}$ & $\begin{array}{c}\text { Fluence } \\
\text { (ions.m }^{-2} \text { ) }\end{array}$ \\
\hline I & 298 & 70 & $1 \times 10^{19}$ & $2.5 \times 10^{21}$ \\
\hline II & 1173 & 70 & $1 \times 10^{19}$ & $4.5 \times 10^{21}$ \\
\hline III & 298 & 8000 & $1.2 \times 10^{17}$ & $1.5 \times 10^{22}$ \\
\hline IV & 1223 & 2000 & $3.3 \times 10^{16}$ & $3.6 \times 10^{19}$ \\
\hline
\end{tabular}


A bright-field TEM image with the associated Select Area Diffraction (SAD) pattern inserted and an EBSD orientation map of a typical UFG and NC tungsten sample used in this study is shown in Figure 1. Ultrafine grains are defined as those having the shortest distance between opposite grain boundaries $<500 \mathrm{~nm}^{12}$ and nanocrystalline grains as having the shortest distance $<100 \mathrm{~nm}^{11}$. As shown in Figure 1, both ultrafine and nanocrystalline grains coexist in the material. EBSD performed on several samples showed $40-50 \%$ of the grains to be high-angle type with grain boundary angles $>15^{\circ}$.

Atomic displacements occur in a material if an energetic particle transfers enough energy to an atom to overcome the displacement threshold energy, $E_{\mathrm{d}}$. The displacement energy for tungsten is reported to be $40 \mathrm{eV} .{ }^{23}$ Assuming a perfectly elastic binary collision, the minimum energy a helium atom, $E_{\min }$, requires to displace a tungsten atom can be calculated by:

$$
E_{\mathrm{min}}=\frac{\left(M_{\mathrm{He}}+M_{\mathrm{W}}\right)^{2}}{4 M_{\mathrm{He}} M_{\mathrm{W}}} \cdot E_{\mathrm{d}}
$$

where $M_{\mathrm{He}}$ and $M_{\mathrm{W}}$ are the masses of the helium and tungsten atoms, respectively. According to equation 1 , a $480 \mathrm{eV}$ helium atom is required to displace a tungsten atom which is in agreement with the literature ${ }^{24}$ of $500 \mathrm{eV}$. In order to explore the role of point defect generation we used helium ions with energies of $70 \mathrm{eV}$ (i.e. below the threshold for atomic displacements in tungsten) and at $2 \mathrm{keV}$ or $8 \mathrm{keV}$ (i.e. above the threshold). 
When vacancies and interstitials are generated in tungsten, their mobility will depend on temperature. Due to their low migration energy of $0.054 \mathrm{eV},{ }^{25}$ interstitials can migrate in tungsten even at RT. Vacancies have a higher migration energy of $1.7 \mathrm{eV} .{ }^{26}$ Therefore higher temperatures are needed for vacancy migration to occur. Different temperatures for tungsten vacancy migration have been reported in literature. Debelle et al. ${ }^{27}$ used positron annihilation spectroscopy and reported that single vacancy migration occurs between 523-573 K. Eleveld and $\mathrm{Veen}^{28}$ used positron annihilation and thermal desorption techniques and reported $650 \mathrm{~K}$ as the temperature were monovacancies migrate to form clusters. However, small vacancy clusters begin to migrate and form cavities at higher temperatures over $773 \mathrm{~K}^{27}$ To explore the effect of vacancy migration, we irradiated at RT (i.e. below the temperature required for vacancy migration) and at $1150 \mathrm{~K}$ or $1223 \mathrm{~K}$ (i.e. above the activation temperature). Helium is known to migrate freely in tungsten even below $\mathrm{RT}^{29,30}$ and will therefore have been mobile at all the temperatures used in this study.

Table 1 illustrates the four experimental regimes compared in this study: I) no atomic displacements $\left(E_{\mathrm{He}}=70 \mathrm{eV}<480 \mathrm{eV}\right)$ and minimal vacancy migration $(T=\mathrm{RT}<600 \mathrm{~K})$; II) no atomic displacements $\left(E_{\mathrm{He}}=70 \mathrm{eV}<480 \mathrm{eV}\right)$ and significant vacancy migration $(T>600 \mathrm{~K})$; III) atomic displacements $\left(E_{\mathrm{He}}>480 \mathrm{eV}\right)$ and minimal vacancy migration $(T=\mathrm{RT}<600 \mathrm{~K})$; and IV) atomic displacements $\left(E_{\mathrm{He}}>480 \mathrm{eV}\right)$ and significant vacancy migration $(T>600 \mathrm{~K})$. For regimes I and II, TEM samples were irradiated ex-situ with $70 \mathrm{eV}$ helium at RT and $1150 \mathrm{~K}$, respectively. For regimes III and IV, the experiments were performed in-situ and the TEM samples were bombarded with $8 \mathrm{keV}$ helium at RT and $2 \mathrm{keV}$ helium at $1223 \mathrm{~K}$, respectively. Despite possible differences in sample thickness, dose rate, incident irradiation angle and penetration depth, comparison of how bubbles are distributed within the grains and the grain 
boundaries is possible and valid since the objective of this work is to qualitatively examine the behavior of helium in this UFG and NC tungsten system and particularly to investigate the irradiation conditions at which preferential bubble nucleation on grain boundaries occurs.

\section{Results and Discussion}

Figure 3 shows bright-field TEM micrographs of UFG and NC tungsten bombarded with 70 $\mathrm{eV}$ helium at RT. The images were taken in under-focus imaging conditions and therefore the bubbles appear bright due to their Fresnel fringes. The images demonstrate a uniform distribution of 2-3 nm bubbles with an average areal-density of approximately 0.04 bubbles. $\mathrm{nm}^{-2}$ at a fluence of $2.5 \times 10^{21}$ ions. $\mathrm{m}^{-2}$. The diffusion coefficient of helium has been reported to be comparable both in the bulk and on grain boundaries. ${ }^{3}$ However, due to the 2D diffusion of helium atoms on the grain boundaries compared to $3 \mathrm{D}$ in the matrix, once helium is captured by a grain boundary it is able to cluster more efficiently. ${ }^{3}$ Similarly, this effect will be even greater on dislocations due to $1 \mathrm{D}$ diffusion. ${ }^{3}$ Since vacancy formation and migration do not occur under these conditions, the nucleation of bubbles can happen when a helium atom binds to an impurity or a thermal vacancy, which can then grow by the addition of more helium atoms. Although in this case $70 \mathrm{eV}$ helium atoms do not displace tungsten atoms, defects could be generated though trap mutation $^{31}$ or loop punching ${ }^{24}$ when more helium atoms trap in a bubble nucleation site (such as a thermal vacancy) thus generating over pressurized bubbles that emit interstitials or dislocation loops to relieve the excess pressure. Bubble formation, however, can also occur due to helium atoms clustering ${ }^{19}$. Figure $3 \mathrm{c}$ shows $3-5 \mathrm{~nm}$ dark spots distributed in the grains. It is expected 
that defects generated from helium-vacancy cluster growth (as described above) can agglomerate and generate dislocation loops. ${ }^{19,32}$ Iwakiri et al.$^{33}$ observed dark spots after low fluence subthreshold $250 \mathrm{eV}$ helium irradiation of coarse grained tungsten and assumed them to be plane agglomerates of implanted helium (i.e. helium platelets). Similar helium platelets have been reported in helium-irradiated molybdenum samples at non-displacement energies $(150 \mathrm{eV}){ }^{34}$ Trinkaus et al. ${ }^{35}$ reported that He platelets formed due to helium irradiation of silicon carbide at RT, can transform into dislocation loops and helium bubbles when subsequently annealed to between $1300 \mathrm{~K}$ and $2100 \mathrm{~K}$. For irradiation conditions below the threshold for atomic displacements in the current study, these dark spots are therefore expected to be caused by strain fields associated with dislocation loops formed due to growing or dissociated platelets or helium platelets themselves. Moreover, formation of helium platelets is expected to occur when vacancy supply is too low (expected at non-displacement energies such as the $70 \mathrm{eV}$ irradiation in this study) for three-dimensional helium-vacancy agglomerations. ${ }^{35}$ The formation of helium platelets can mark the initial stage of bubble formation. ${ }^{36,37}$ At high temperatures ${ }^{34}$ or high helium doses ${ }^{34}$ these platelets can evolve into small bubbles. It has been reported that under high internal pressure, several small bubbles are a lower energy configuration than one single large bubble. $^{38}$

It might be expected that the grain boundaries, which are effective helium traps, ${ }^{39}$ will become regions of high helium concentration. Surprisingly, in the sub-threshold RT irradiations, neither large bubble formation on the boundary nor denuded zones around the grain boundaries were observed despite the high fluence of $2.5 \times 10^{21}$ ions. $\mathrm{m}^{-2}$.

Figure 4 shows TEM micrographs of UFG and NC tungsten bombarded with $70 \mathrm{eV}$ helium at $1173 \mathrm{~K}$. Similar to the RT irradiation at $70 \mathrm{eV}$, a uniform distribution of approximately $3 \mathrm{~nm}$ 
bubbles with an areal density of approximately 0.025 bubbles. $\mathrm{nm}^{-2}$ (at a fluence of $4.5 \times 10^{21} \mathrm{~m}^{-2}$ ) were observed with defects assumed to be helium platelets or associated dislocation loops as discussed above. At this elevated temperature, vacancy migration occurs but the creation of vacancies under these irradiation conditions is limited to thermal vacancies or vacancies generated through possible trap mutation processes during bubble growth (although such vacancies are by definition immediately incorporated into the generating bubble). As well as grain boundaries being efficient vacancy sinks, Bai et al. ${ }^{5}$ showed that interstitials absorbed by grain boundaries can be re-emitted to combine with nearby vacancies. The removal of vacancies from the regions surrounding the grain boundaries could lead to a reduction in the density of potential bubble nucleation sites. However, in this experiment bubbles were uniformly distributed across the grains. Recently, Sefta et al. ${ }^{19}$ demonstrated through molecular dynamic simulations that a $\{100\}$ tungsten surface intersected by a grain boundary retains around $20 \%$ more helium than $\{100\}$ single-crystal surface at $1200 \mathrm{~K}$ when irradiated with $60 \mathrm{eV}$ helium ions. Based on that work, one might expect to observe larger bubbles on the grain boundaries than the matrix. However, this was not observed in the current study under these sub-threshold irradiation conditions.

Comparing the $70 \mathrm{eV}$ experiments at RT and $1173 \mathrm{~K}$, no large differences were observed. The higher temperature experiment required a greater final fluence $\left(4.5 \times 10^{21} \mathrm{~m}^{-2}\right.$ versus $2.5 \times 10^{21} \mathrm{~m}^{-2}$ for the RT experiment) to produce comparable bubble size to those formed at lower temperatures. This can possibly be explained by considering vacancy migration, the probability of helium trapping and the proximity of the surface. Although vacancies are not generated via atomic collisions in the $70 \mathrm{eV}$ case, those which are naturally present in the relatively-shallow implanted region will be mobile at $1173 \mathrm{~K}$ and will be able to migrate to the surface. Sefta et al. 
demonstrated how helium retention in the tungsten matrix decreases with increasing temperature. ${ }^{19}$ If this process (helium migration to the surface) had a greater effect than the similarly-increased thermal vacancy production rate, then it will have lowered the number of bubble nucleation sites meaning a helium atom is more likely to escape to a surface or grain boundary before it is trapped in the matrix. Therefore the bubbles were smaller and had a lower areal-density than at the same fluence in the lower temperature experiments.

Figure 5 shows TEM micrographs of UFG and NC tungsten bombarded with $8 \mathrm{keV}$ helium at RT during an in-situ experiment. At this energy, vacancies and interstitials are generated by atomic collisions; however the vacancies are immobile at RT. These conditions resulted in bubbles of approximately $5 \mathrm{~nm}$ in diameter, uniformly distributed and with an areal density of approximately 0.01 bubbles. $\mathrm{nm}^{-1}$ at a fluence of $1.5 \times 10^{22}$ ions. $\mathrm{m}^{-2}$. Irradiation with sufficient energy to induce atomic displacements and at a temperature $(1223 \mathrm{~K})$ sufficient to make vacancies mobile, drastically changed the resulting microstructure compared to the other experimental regimes, as shown in Figure 6. At this higher temperature, vacancies and interstitials are generated due to atomic collisions and both are able to migrate. Bubbles of varying size were uniformly distributed across the grains but with lower areal-densities on the smaller nanocrystalline grains. As shown in Figures 6 and 7, defects on ultrafine grains and a lower areal-density of bubbles on the nanocrystalline grains were observed. The grain boundaries of the nanocrystalline grains were decorated with large and facetted cavities. These have been reported to occur due to high vacancy supply and anisotropic surface energies at high temperatures. $^{40}$ The variation of bubble size and density among different grains in the $2 \mathrm{keV}$ and $1123 \mathrm{~K}$ irradiation case at a fluence of $3.6 \times 10^{19}$ ions.m ${ }^{-2}$ is illustrated in Figure 8 . It should be noted that similar imaging conditions were used so that the average bubble size is not 
significantly affected by imaging conditions. Grains of less than $\sim 60 \mathrm{~nm}$ showed lower bubble densities but higher bubble sizes than other nanocrystalline grains $(60-100 \mathrm{~nm})$ and ultrafine grains $(100-500 \mathrm{~nm}) .{ }^{14}$ While the high density of bubbles on the grain boundaries of these materials (Figure 6) demonstrates better helium sink efficiency, degradation of mechanical properties can possible as a result of large cavity formation. Other structural nuclear materials were shown to suffer, under stress from high temperature mechanical property degradation (ductility deterioration, creep and fatigue life reduction, embrrittlement ) due to bubble growth on grain boundaries which can initiate crack formation and intergranular brittle failure. ${ }^{41}$ In addition to bubble formation, it has also been reported that the formation of defect clusters, dislocations and dislocation loops occurs in ultrafine grains at high temperatures due to the increased mobility of defects. ${ }^{14}$ In both experiments in which the energy of the incident helium was above that required to cause atomic displacements, irradiation enhanced and/or induced diffusion are expected. ${ }^{42}$ However, defect formation and migration ${ }^{14}$ were much more pronounced in the higher temperature experiment suggesting the thermal enhancement to diffusion was a more significant factor. It should also be mentioned that in both experiments (2 $\mathrm{keV}$ and $8 \mathrm{keV}$ irradiations), no significant modification of the thin edges of the TEM samples was observed during the in situ irradiations so therefore sputtering is not considered to have been a relevant factor.

One remaining question is whether the facetted cavities in the high temperature experiments were helium bubbles (formed by helium-vacancy agglomeration) or voids (formed by vacancy clustering) or started as small bubbles and ended up as a large faceted voids. This issue can be explored by examining the bubble density and distribution in the nanocrystalline grains in the high temperature experiment ( $2 \mathrm{keV}$ irradiation at $1223 \mathrm{~K}$ ) compared to the low temperature case 
( $8 \mathrm{keV}$ irradiation at RT). The two experiments were run at different conditions with helium diffusion and escape as well as bubble size and pressure all potentially different. However, in the low temperature experiment, the bubbles were uniformly distributed over each grain with a relatively-high areal-density of approximately 0.01 bubbles.nm ${ }^{-2}$ at a fluence of $1.5 \times 10^{21}$ ions. $^{-}$ ${ }^{2}$ regardless of the grain size. Conversely in the high temperature experiment, bubbles were more spatially separated with a bubble density of approximately 0.003 bubbles. $\mathrm{nm}^{-2}$ in the ultrafine grains at a fluence of $3.6 \times 10^{19}$ ions.m ${ }^{-2}$. No bubble coalescence, which could alter the bubble density, was observed during the in situ irradiations. The lower areal density in the high temperature experiment suggest that the helium atoms were able to travel greater distances before being trapped and so will have reached grain boundaries in greater numbers. Therefore the facetted cavities on the grain boundaries are likely to be large bubbles given this high flux of helium.

Comparison of the $70 \mathrm{eV}$ and $2 \mathrm{keV}$ helium irradiations at 1173 and $1223 \mathrm{~K}$, respectively, reveals the importance of point defect production for the formation of large bubbles on grain boundaries. As shown in Fig. 6, under displacing $2 \mathrm{keV}$ helium irradiation, large bubbles were formed on grain boundaries at the end-fluence of $4.0 \times 10^{20}$ ions. $\mathrm{m}^{-2}$. However, under $70 \mathrm{eV}$ helium irradiation which cannot create atomic displacements, no such large bubbles were observed on the grain boundaries as shown in Fig. 4 despite the higher end-fluence of $4.5 \times 10^{21}$ ions. $\mathrm{m}^{-2}$ versus and comparable irradiation temperatures. Vacancies may also enhance bubble growth by increasing the mobility of helium through the matrix in the form of heliumvacancy complexes, which are expected to form ${ }^{43}$ and migrate ${ }^{33}$ under these conditions. Once in the boundaries, both the helium and vacancies are able to migrate and agglomerate to form bubbles. However, in the absence of an irradiation-induced vacancy supply in the $70 \mathrm{eV}$ case, 
helium atoms which reach a grain boundary can only become immobilized in a pre-existing region of low electron-density on the boundary, combine with a thermal vacancy or continue to migrate on the boundary until they reach a surface and escape. It is then, concluded that both vacancy generation and migration are necessary to efficiently trap helium on grain boundaries as summarized in Table 2. These results indicate the importance of helium, vacancy, and possibly helium-vacancy cluster formation and the subsequent migration to grain boundaries in the irradiation response of UFG and NC tungsten.

\section{Table 2: Output results summary from this work}

\begin{tabular}{|c|c|c|c|c|c|c|}
\hline \multirow[b]{2}{*}{ Regime } & \multirow{2}{*}{$\begin{array}{c}\text { Sample } \\
\text { temperature } \\
(K)\end{array}$} & \multirow[b]{2}{*}{$\begin{array}{c}\text { Energy } \\
(e V)\end{array}$} & \multicolumn{3}{|c|}{ Defect dynamics } & \multirow{2}{*}{$\begin{array}{c}\text { Preferential } \\
\text { nucleation of } \\
\text { bubbles on gbs }\end{array}$} \\
\hline & & & Displacement & $\begin{array}{l}\text { Vacancy } \\
\text { migration }\end{array}$ & $\begin{array}{l}\text { Interstitial } \\
\text { migration }\end{array}$ & \\
\hline I & 298 & 70 & No & No & Yes & No \\
\hline II & 1173 & 70 & No & Yes & Yes & No \\
\hline III & 298 & 8000 & Yes & No & Yes & No \\
\hline IV & 1223 & 2000 & Yes & Yes & Yes & Yes \\
\hline
\end{tabular}

It should be noted that the $70 \mathrm{eV}$ experiments have higher fluxes ( $\sim 300$ and $\sim 80$ times the flux in the 2 and $8 \mathrm{keV}$ experiments respectively) and very low implantation depth. Surface effects (helium escape to the surface) and rate effects (difference in helium implantation rate) can be a concern. Although the $70 \mathrm{eV}$ experiments have higher implantation rate and mutual clustering of helium can occur, the flux is still much ( 1000 times) lower than the expected helium flux for surface saturation and fuzz formation ${ }^{9,8}$, and therefore, helium can diffuse to grain boundaries. Moreover, MD simulations by Sefta et al. ${ }^{19}$ demonstrated grain boundary effects at even higher fluxes $\left(2.5 \times 10^{27}\right.$ ions. $\left.\mathrm{m}^{-2} \cdot \mathrm{s}^{-1}\right)$. 
While the low penetration depth of the $70 \mathrm{eV}$ experiments can lead to higher helium escape to the surface (lower retention), helium particles implanted in the proximity of a grain boundary should be able to diffuse to the grain boundary considering the low migration energy of helium in tungsten $(\sim 0.24 \mathrm{eV})$ and the corresponding very high diffusion rate ${ }^{44}$. In addition, heliumvacancy complex formation should enable helium diffusion to nearby grain boundaries thought complex migration in the high temperature experiment $(70 \mathrm{eV}$ and $1173 \mathrm{~K})$. The recent simulations of Sefta et al. ${ }^{19}$ was performed at $60 \mathrm{eV}$ and helium implantation percentage was $85 \%$ in tungsten with a grain boundary at temperatures ranging from 500-2000K. Moreover, bubbles were shown to form at the area deeper than that of the helium implantation determined by SRIM at non-displacement energies (eg. $50 \mathrm{eV}) .{ }^{16}$ demonstrating larger diffusion lengths of helium than expected. Stereoscopic measurements and thermal desorption spectroscopy measurements (not available in this work) should help in addressing concerns regarding surface and rate effects.

\section{Summary}

Ultrafine grained and nanocrystalline tungsten TEM samples have been irradiated under different helium energy and temperature combinations to investigate the radiation tolerance to bubble formation and the role of grain boundaries in trapping helium. Bubbles were shown to nucleate uniformly at energies below the displacement threshold of tungsten regardless of temperature. Bombarding with helium energies over the displacement threshold, demonstrated more bubbles or helium trapping on the grain boundaries only at high temperatures at which vacancies are mobile. It is concluded that at the experimental conditions used in this work, vacancy generation and migration are necessary conditions for enhanced trapping of helium at 
grain boundaries demonstrating the importance of these phenomena for the radiation response of tungsten materials with tailored grain size. Future work will focus on stereoscopic and thermal desorption spectroscopy measurements to support these findings and eliminate doubts about surface and rate effects.

\section{Acknowledgments}

O. El-Atwani would like to thank Prof. Jean Paul Allain for his help in funding him while performing part of the work. K. Hattar acknowledges the Division of Materials Science and Engineering, Office of Basic Energy Sciences, U.S. Department of Energy. Sandia National Laboratories is a multi-program laboratory managed and operated by Sandia Corporation, a wholly owned subsidiary of Lockheed Martin Corporation, for the U.S. Department of Energy's National Nuclear Security Administration under contract DE-AC04-94AL85000. Experimental work using the MIAMI facility was supported by the UK's Engineering and Physical Research Council under grant number EP/H018921/1.

\section{Figure Captions:}

Figure 1: (a) Bright-field TEM micrograph of an UFG and NC tungsten sample and (b) EBSD map of a sample demonstrating the presence of high-angle grain boundaries in most regions of the sample.

Figure 2: Summary of the four experimental regimes compared in the current study.

Figure 3: Bright-field TEM micrographs of UFG and NC tungsten irradiated with $70 \mathrm{eV}$ helium ions (flux of $1 \times 10^{19}$ ions. $\mathrm{m}^{-2} . \mathrm{s}^{-1}$ ) to a fluence of $2.5 \times 10^{21}$ ions. $\mathrm{m}^{-2}$ at RT demonstrating: (a) uniform distribution of bubbles; (b) no denuded zones along the grain boundaries; (c) defects assumed to helium platelets or associated dislocation loops (see text for discussion); and (d) coexistence of helium platelets or dislocation loops and 2-3 nm bubbles.

Figure 4: Bright-field TEM micrographs of UFG and NC tungsten irradiated with $70 \mathrm{eV}$ helium ions (flux of $1 \times 10^{19}$ ions. $\mathrm{m}^{-2} \cdot \mathrm{s}^{-1}$ ) to $4.5 \times 10^{21}$ ions. $\mathrm{m}^{-2}$ at $1173 \mathrm{~K}$ demonstrating: (a) uniform areal-distribution of bubbles; (b) no denuded zones near the grain boundaries and uniform arealdistribution of bubbles on an ultrafine grain with few helium platelets observable. 
Figure 5: Bright-field TEM micrographs of UFG and NC tungsten irradiated with $8 \mathrm{keV}$ helium (flux of $1.2 \times 10^{17}$ ions. $\mathrm{m}^{-2} . \mathrm{s}^{-1}$ ) to a fluence of $1.5 \times 10^{22}$ ions.m $\mathrm{m}^{-2}$ at RT demonstrating: (a) uniform distribution of bubbles and no denuded zones near grain boundaries; and (b) same region as (a) in an over-focused imaging condition with bubbles appearing dark.

Figure 6: Bright-field TEM micrographs of UFG and NC tungsten irradiated with $2 \mathrm{keV}$ helium ions (flux of $3.3 \times 10^{16}$ ions. $\mathrm{m}^{-2} . \mathrm{s}^{-1}$ ) at $1223 \mathrm{~K}$ demonstrating: (a) overview of sample with bubbles decorating grain boundaries at a fluence of $3.6 \times 10^{19}$ ions. $^{-2}$; (b) nanocrystalline grain with large facetted bubbles/voids on grain boundaries and few bubbles in the grain matrix at a fluence of $3.6 \times 10^{19}$ ions. $\mathrm{m}^{-2}$; and (c) grain boundary and (d) grain boundary triple-junction decorated by facetted bubbles with different sizes inside ultrafine grains at fluence of $4.0 \times 10^{20}$ ions. $\mathrm{m}^{-2}$.

Figure 7: Bright-field TEM micrograph of UFG irradiated with $2 \mathrm{keV}$ helium ions at $1223 \mathrm{~K}$ to a fluence of $3.6 \times 10^{19}$ ions. $\mathrm{m}^{-2}$ demonstrating uniform distribution defect clusters, dislocations and bubbles.

Figure 8: Bubble areal density (right columns) and average bubble size (left columns) vs grain size for $2 \mathrm{keV}$ helium ions irradiation at $1223 \mathrm{~K}$ and a fluence of $3.6 \times 10^{19}$ ions. $\mathrm{m}^{-2}$. Bubbles located on grain boundaries were not counted. A total of 18 neighboring grains were analyzed in order to ensure maximum consistency in ion fluence, sample thickness and irradiation temperature.

\section{References}

${ }^{1}$ T.D. Shen, S. Feng, M. Tang, J.A. Valdez, Y. Wang, K.E. Sickafus, Appl. Phys. Lett. , 90 (2007) 263115.

${ }^{2}$ M. Samaras, P. M. Derlet, H. Van Swygenhoven,M. Victoria, Philos. Mag. 83, (2003) 3599

${ }^{3}$ B.N. Singh, T. Leffers, W.V. Green, M. Victoria, J. Nucl. Mater., 125 (1984) 287-297.B. 847

${ }^{4}$ C. Sun, M. Song, K.Y. Yu, Y. Chen, M. Kirk, M. Li, H. Wang, and X. Zhang, Metallurgical and Materials Transactions A, 44 (2013) 1966-1974.

${ }^{5}$ X-M. Bai, A. F. Voter, R.G. Hoagland, M. Nastasi, B.P. Ulberuaga, Science 327 (2010) 1631

${ }^{6}$ B. Lipschultz et al., Nucl. Fusion, 47 (2007) 1189-1205.

${ }^{7}$ O. El-Atwani, M. Efe, B. Heim, J. P. Allain, J. Nucl. Mater., 434 (2013) 170-177

${ }^{8}$ S. Kajita, W. Sakaguchi, N. Ohno, N. Yoshida, T. Saeki, Nucl. Fusion, 49 (2009) 095005.

${ }^{9}$ M.J. Baldwin, R.P. Doerner, J. Nucl. Mater., 404 (2010) 165-173

${ }^{10}$ G. Federici, C.H. Skinner, J.N. Brooks, J.P. Coad, C. Grisolia, A.A. Haasz, A. Hassanein, V. Phlipps, C.S. Pitcher, J. Roth, W.R. Wampler, D. G. Whyte, Nuclear Fusion. 41 (2001) 1967 
${ }^{11}$ Q. Wei, H.T. Zhang, B.E. Schuster, K.T. Ramesh, R.Z. Valiev, L.J. Kecskes, R.J. Dowding, L. Magness, K. Cho, Acta Mater., 54 (2006) 4079-4089

${ }^{12}$ Q. Wei, T. Jiao, K.T. Ramesh, E. Ma, L.J. Kecskes, L. Magness, R. Dowding, V.U. Kazykhanov, R.Z. Valiev, Acta Mater., 54 (2006) 77-87

${ }^{13}$ M. Demkowicz, M.J. Misra, A. Robertson, I.M. Robertson, R.G. Hoagland, Scripta Materialia ${ }^{14}$ O. El-Atwani, J.A. Hinks, G. Greaves, S. Gonderman, T. Qiu M. Efe, J.P. Allain, Sci. Rep. 4 (2014) 4716

${ }^{15}$ O. El-Atwani, M. Efe, B. Heim, J. P. Allain, J. Nucl. Mater., 434 (2013) 170-177

${ }^{16}$ S. Kajita, N. Yoshida, R. Yoshihara, N. Ohno, M. Yamagiwa, J. Nucl. Mater, 418 (2011) 152158.

${ }^{17}$ P. Scott, J. Nucl. Mater., 211 (1994) 101-122

${ }^{18}$ M.Efe, O. El-Atwani, Y. Guo, D. Klenosky, Scripta Materialia 70 (2014) 31-34

${ }^{19}$ S. Sefta, K. D. Hammond, N. Juslin, B.D. Wirth, Nucl. Fusion 53 (2013) 073015

${ }^{20} \mathrm{~K}$. Hattar et al. NIMB submitted

${ }^{21}$ J.P. Allain, M. Nieto, M.R. Hendricks,P. Plotkin, S.S. Harilal, A. Hassanein, Review of Scientific Instruments, 78(11), (2007) 113105.

${ }^{22}$ J.F. Ziegler, M.D. Ziegler, J.P. Biersack, Nuclear Instruments and Methods in Physics

Research B 268 (2010) 1818-1823

${ }^{23}$ Jung P. 1991 Atomic Defects in Metals (Landolt-Bornstein New Series III/25) ed H. Ullmaier (Berlin: Springer).

${ }^{24}$ Y. Minyou, Plasma Sci. Technol., 7 (2005) 2828-2834.

${ }^{25}$ F. Dausinger, H. Schultz, Phys. Rev. Lett. 35 (1975) 1773.

${ }^{26}$ R.W. Balluffi, J. Nucl. Mater. 69-70 (1978) 240.

${ }^{27}$ A. Debelle, M.F. Barthe, T. Sauvage. J. Nucl. Mater, 376 (2008) 216-221

${ }^{28}$ H. Eleveld, A. van Veen, J. Nucl. Mater, 212-215 (1994) 1421-1425

${ }^{29}$ X. Shu, P. Tao, X. Li, Y. Yu, Nuclear Instruments and Methods in Physics Research B 303 (2013) 84-86

${ }^{30}$ A. Wagner, D.N. Seidman, PRL 42 (1979) 515-518

${ }^{31}$ L.M. Caspers, R.H.J. Fastenau, A. Van Veen, W.F.W.M. Van Heugten, Phys. Stat. Sol. (a) 46 (1978) 541

${ }^{32}$ E. Olieviero, M.F. Beaufort, J.F. Barbot, J. Appl. Phys 90 (2001) 1718-1724

${ }^{33}$ H. Iwakiri, K. Yasunaga, K. Morishita, N. Yoshida, J. Nucl. Mater., 283-287 (2000) 11341138

${ }^{34}$ J.H.Evans, A. Van veen, L.M. Caspers, Nature 291 (1981) 310-312

${ }^{35}$ H. Trinkaus, B.N. Singh, J. Nucl.Mater, 323 (2003) 229-242

${ }^{36}$ P. F. P. Fichtner, J. R. Kaschny, R. A. Yankov, A. Mu"cklich, U. Kreißig, and W. Skorupa, Appl. Phys. Lett. 70 (1997) 732

${ }^{37}$ E. Oliviero, M. F. Beaufort, J. F. Barbot, J. Appl. Phys., 90, (2001) 1718-1724

${ }^{38} 8$ M. W. Finnis, A. Van Veen, and L. M. Caspers, Radiat. Eff. 78, (1983) 121

${ }^{39}$ B.N. Singh, T. Leffers, W.V. Green, M. Victoria, J. Nucl. Mater., 125 (1984) 287-297.

${ }^{40}$ K. Niwase, T. Ezawa, F.E. Fujita, H. Kusanagi, H. Takaku, Rad. Eff. 106 (1988) 65-76

${ }^{41}$ H. Ullmaier, Nuclear Fusion 24, (1984) 1039-1083

${ }^{42}$ J. Delafond, C. Jaouen, J.P. Rivière, C. Fayoux, Mater. Sci. Eng., 69 (1985) 117-121.

${ }^{43}$ H. T. Lee, A. A. Haasz, J.W. Davis, R.G. Macaulay-Newcombe, J.Nucl.Mater 360 (2007) 196207 
${ }^{44}$ J. Amano, D. N. Seidman, J. Appl. Phys. 56 (4) (1984) 982-992 

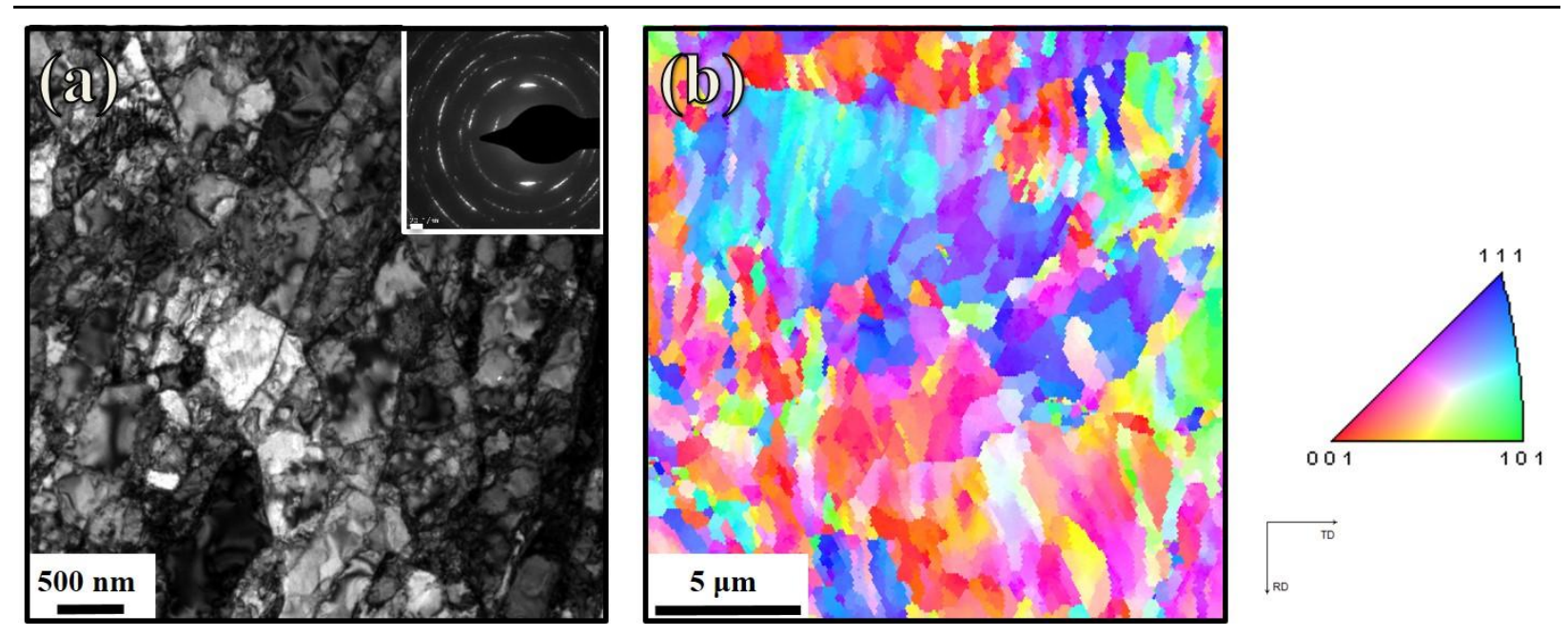


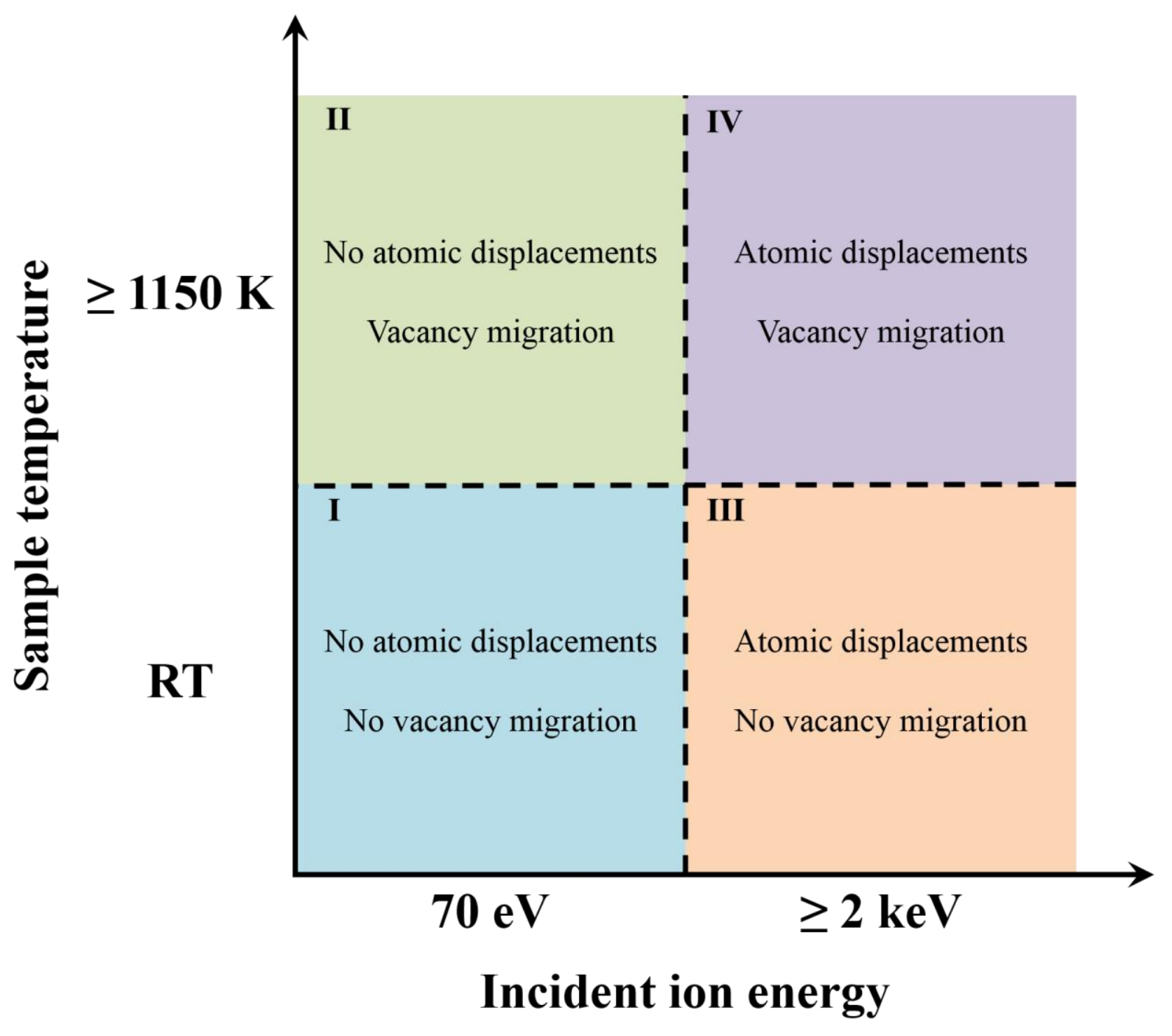



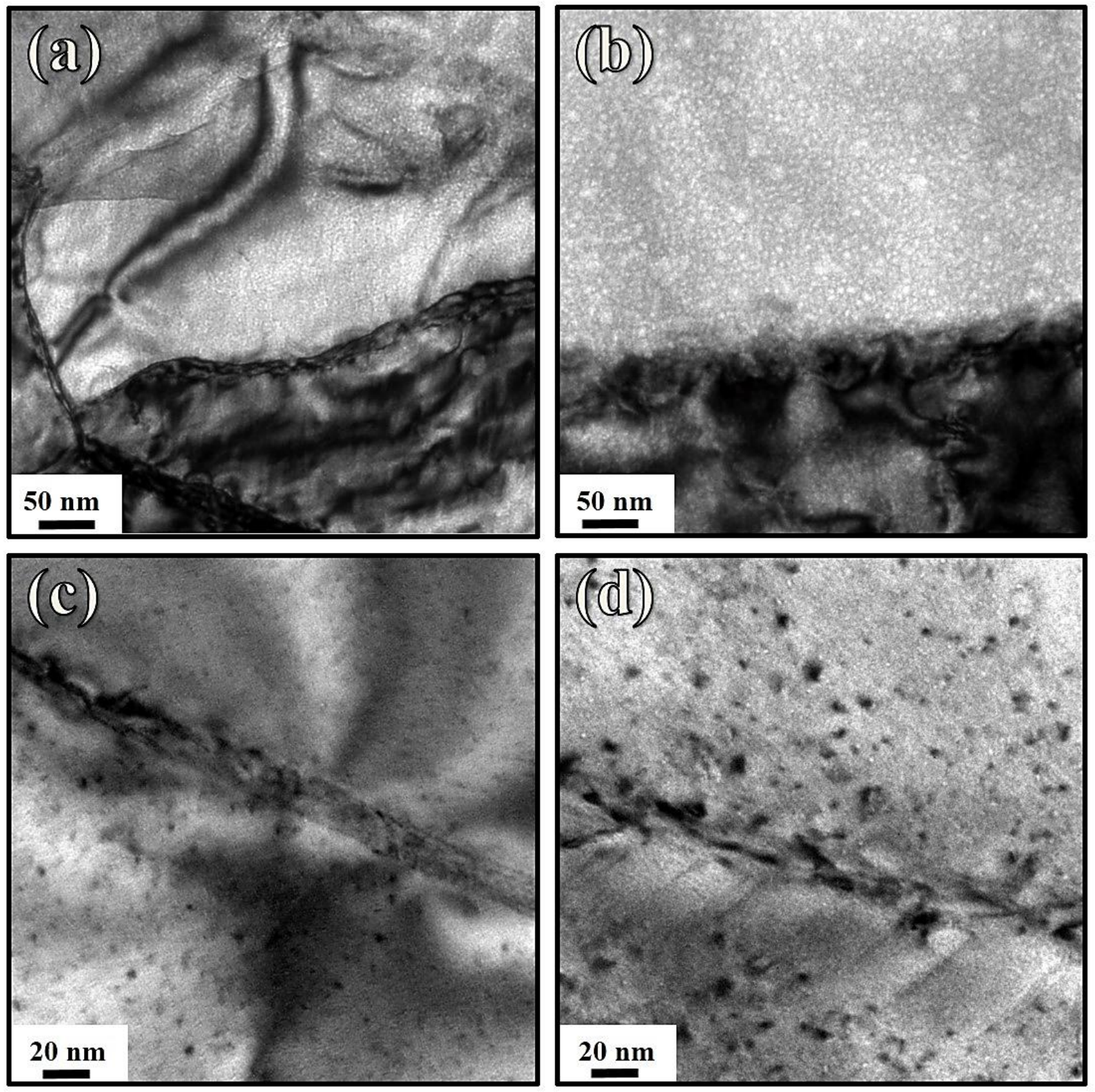

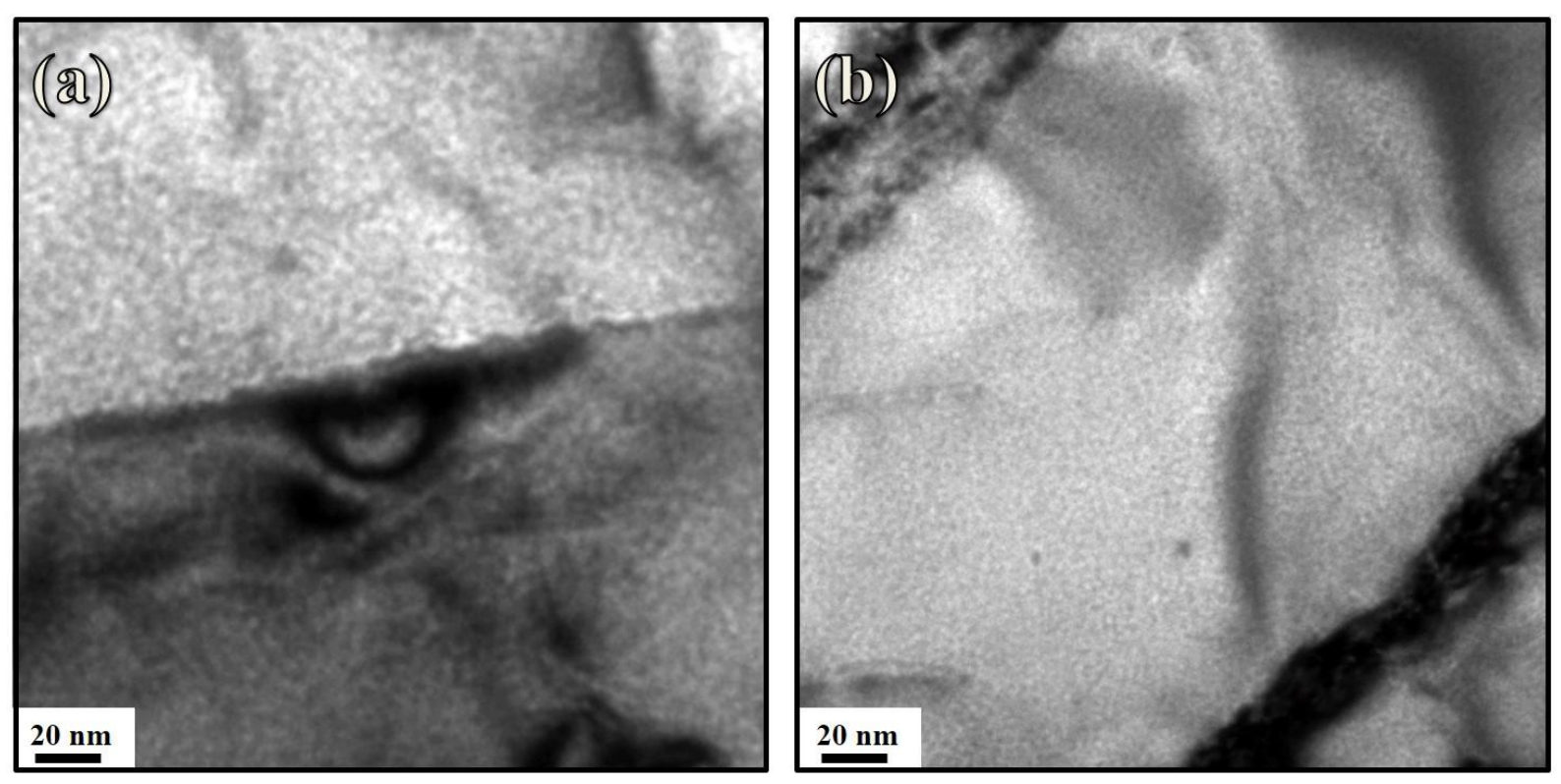

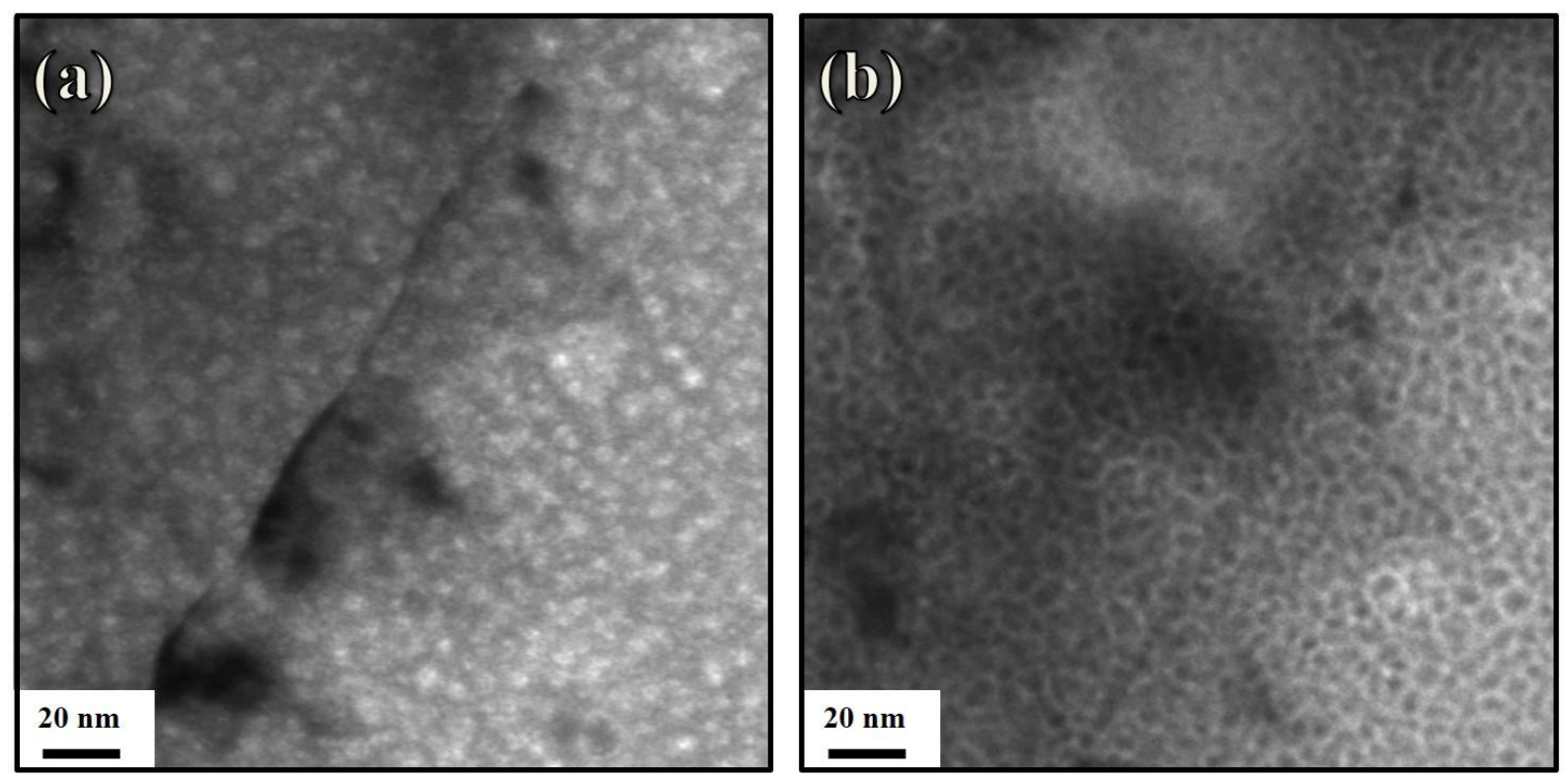

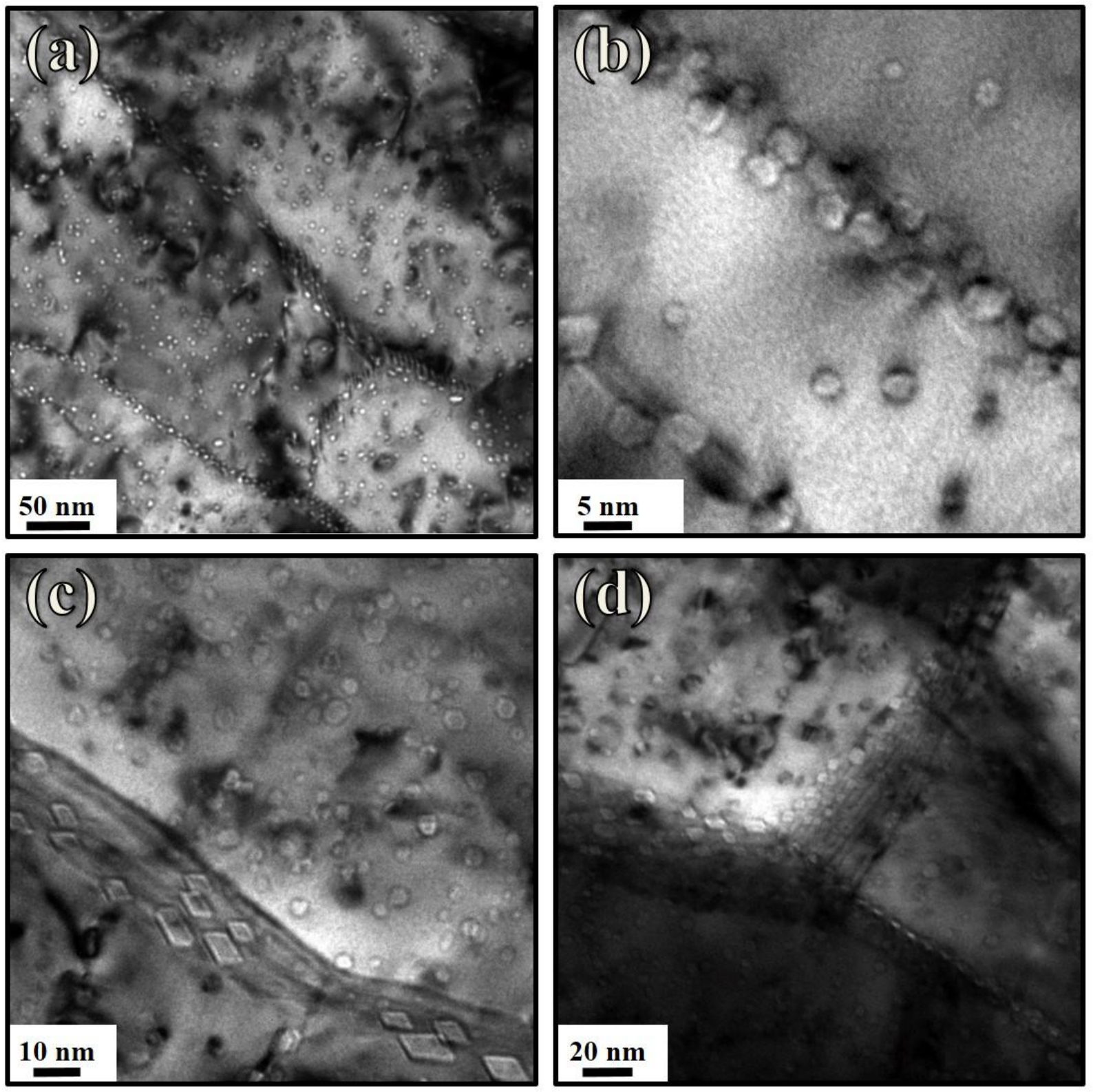


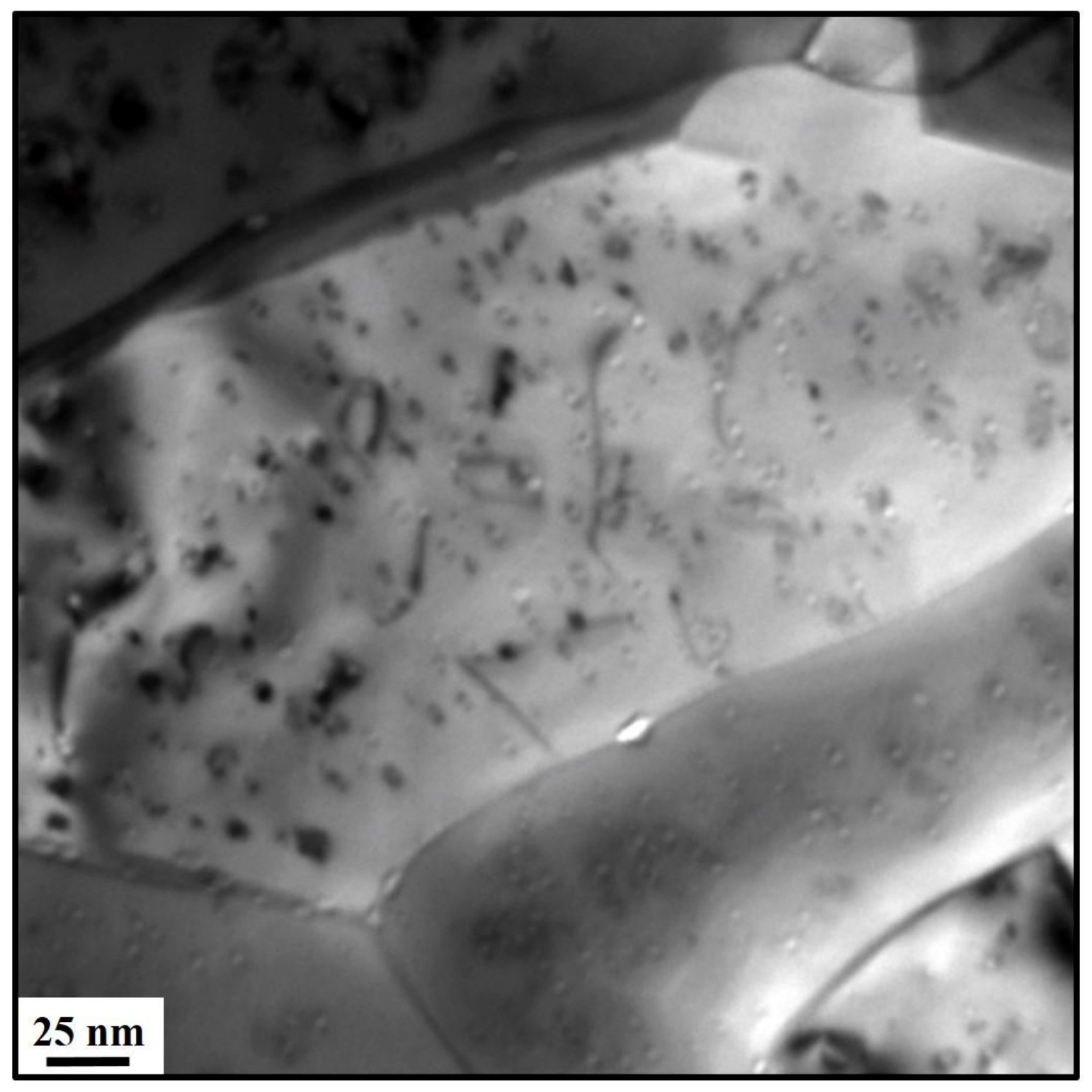




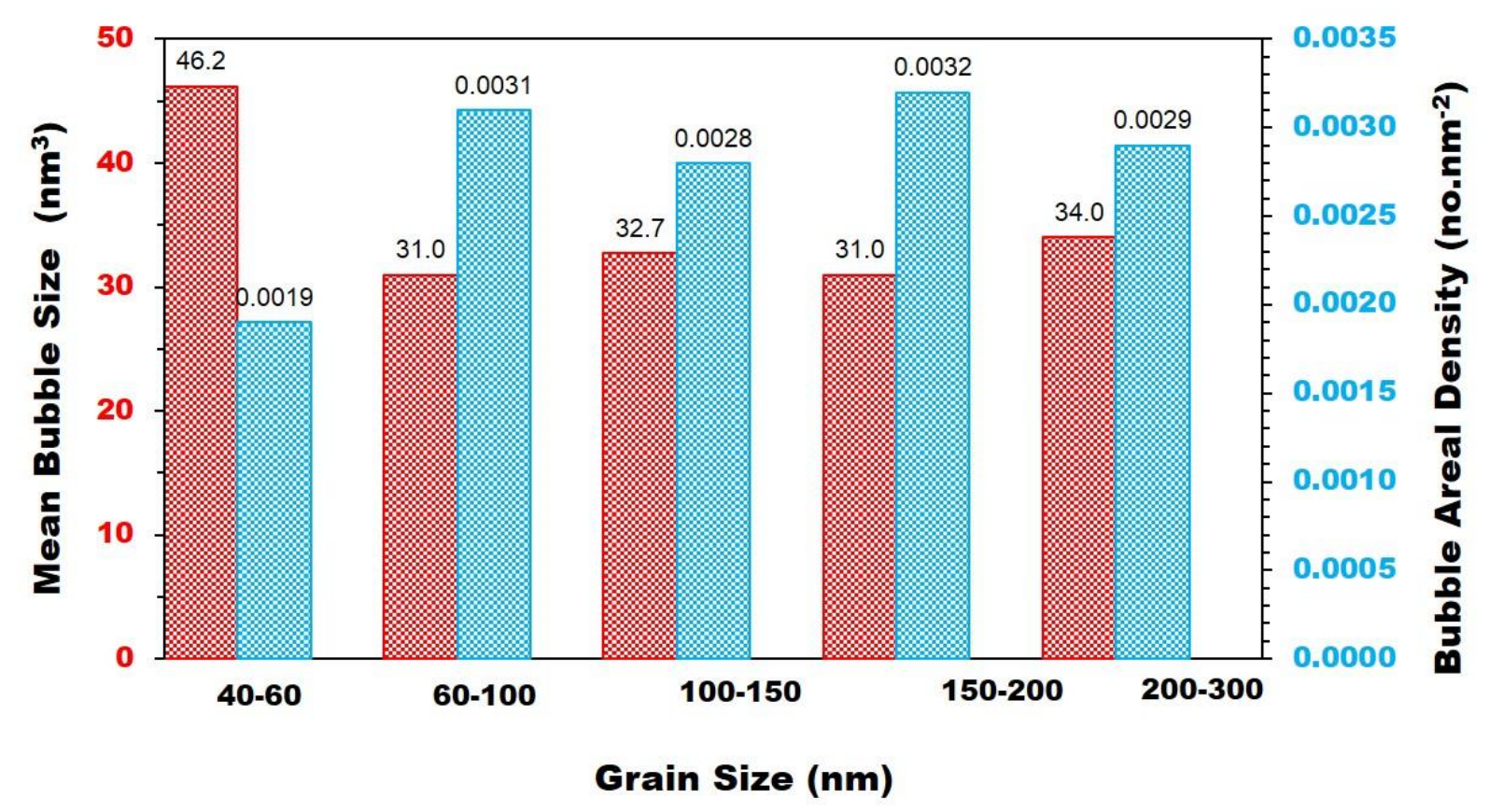



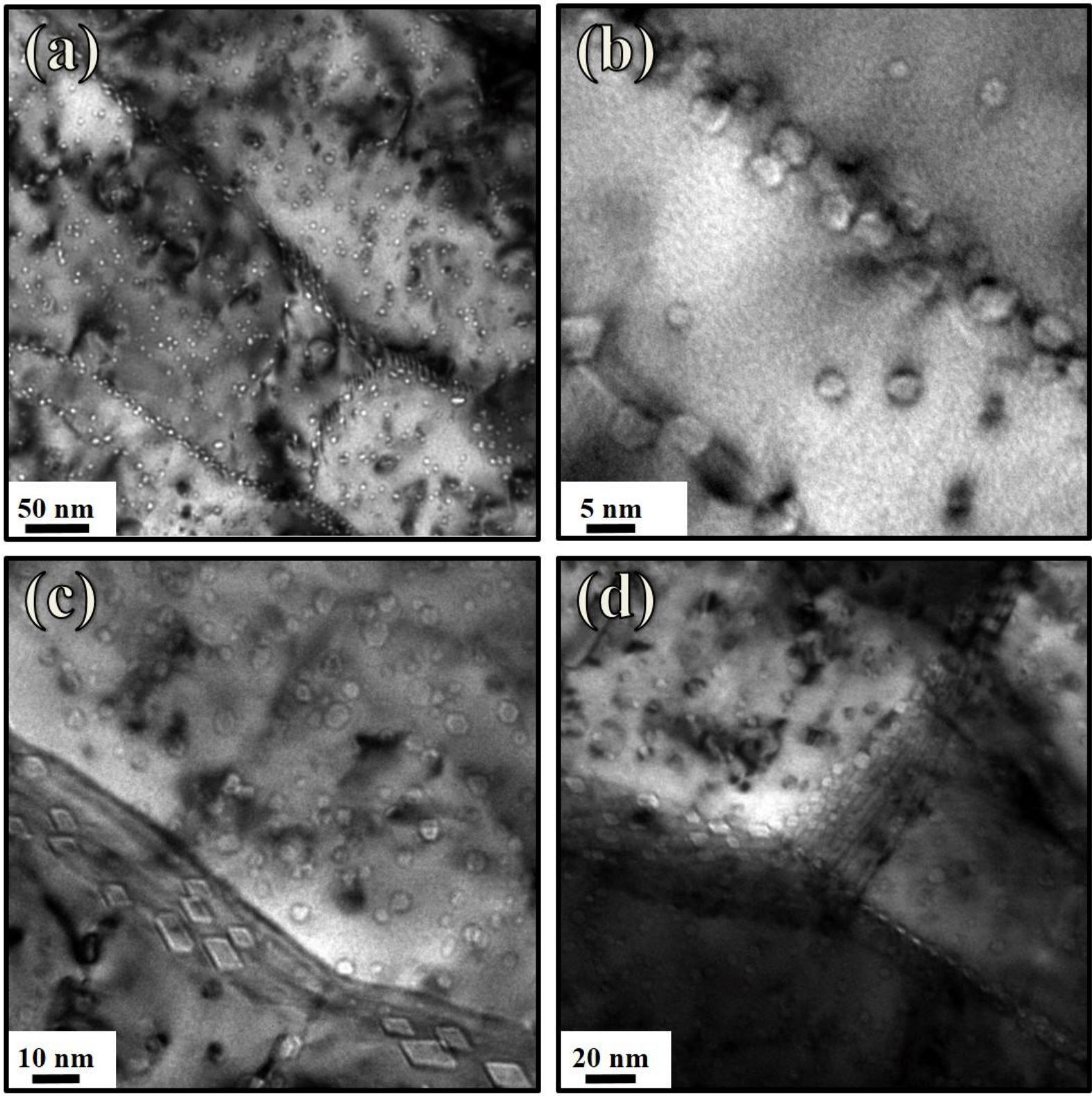

Bright-field TEM micrographs of UFG and NC tungsten irradiated with $2 \mathrm{keV}$ helium ions (flux of $3.3 \times 10^{16}$ ions. $\mathrm{m}^{-2} \cdot \mathrm{s}^{-1}$ ) at $1223 \mathrm{~K}$ demonstrating: (a) overview of sample with bubbles decorating grain boundaries at a fluence of $3.6 \times 10^{19}$ ions. $\mathrm{m}^{-2}$; (b) nanocrystalline grain with large facetted bubbles/voids on grain boundaries and few bubbles in the grain matrix at a fluence of $3.6 \times 10^{19}$ ions. $\mathrm{m}^{-2}$; and (c) grain boundary and (d) grain boundary triple-junction decorated by facetted bubbles with different sizes inside ultrafine grains at fluence of $4.0 \times 10^{20}$ ions. $\mathrm{m}^{-2}$. 